\title{
Structure and Properties of Fatigued Segmented Poly(urethaneurea)s II. Structural Analyses of Fatigue Mechanism
}

\author{
Mitsuhiro Shibayama, Yuichi OHKI, Tetsuo Kotani, \\ and Shunji NOMURA \\ Department of Polymer Science and Engineering, Kyoto Institute of Technology, \\ Matsugasaki, Sakyoku, Kyoto 606, Japan
}

(Received December 4, 1986)

\begin{abstract}
The fatigue mechanism of segmented poly(urethaneurea)s due to a sinusoidal strain was investigated from the structural point of view by means of small angle light scattering (SALS), small angle X-ray scattering (SAXS), and infrared dichroism (IRD) and was compared with the uniaxial deformation mechanism. The SALS patterns of the original sample films indicated the existence of a spherulite texture. There appeared a ring diffraction pattern in the SAXS photograph for the as-cast sample. The diffraction pattern came to have azimuthal angular dependence when stretched or fatigued; which was characterized by a defect in intensity in the stretching direction and a local concentration in intensity at the edge of the defect. Those indicated a selective destruction of the hard segment domain in the equatorial zone of the spherulite. The fatigued mechanism is proposed in order to account for the experimental results based on the spherulite deformation model. It was also found that samples having longer soft segments have higher degree of phase separation at the beginning and that these mechanical properties and orientational behavior are less sensitive to fatigue time.
\end{abstract}

KEY WORDS Poly(urethaneurea) / Fatigue Mechanism / Spherulite

Deformation / Infrared Dichroism / Small Angle X-Ray Scattering / Small

Angle Light Scattering /

Segmented poly(urethaneurea)s (SPUU) and segmented poly(urethane)s (SPU) have been considered for use as high performance elastomeric materials. The unique mechanical properties of SPUU and SPU result from their heterogeneous microdomain structure composed of the hard and the soft segments. Hydrogen bonding also plays an important role in its properties. Structures of SPUU has been extensively studied in terms of DSC, infrared dichroism (IRD), small angle X-ray (SAXS) and neutron scattering (SANS) techniques. ${ }^{1-7}$ Kimura et al..$^{1,5}$ utilized small angle light scattering (SALS), SAXS, and IRD and studied the deformation mechanism of SPUU. They found a spherulitic structure in SPUU and explained the deformation mechanism of
SPUU by the analogy of the deformation mechanism of crystalline polymers, e.g., polyethylene. Bonart et al ${ }^{8,9}$ proposed a different model of deformation on segmented poly(urethane) (SPU), which is based on rotation of a particle having an anisotropic shape in a flow field (domain orientation) followed by segment orientation. The deformation mechanism of SPUU and/or of SPU is, however, still an open question. This fact is partially due to the complexity of the microdomain structure of SPUU and SPU. It is clear that the deformation mechanism depends on the chemical structure of SPUU or SPU. Desper et al. ${ }^{10}$ proposed three possible models of deformation: a shear model, a tensile model, and a rotation or translation of inde- 
pendent particles, depending on the chemical structure of the poly(urethane). Those models except for the spherulite model, however, are proposed based on analyses which are restricted in segment order (sub-nanometers) and/or lamella order (a few tens of nanometers). Therefore it is not clear whether those models can be applied to explain changes of superstructures (microns order) due to stretching.

The fatigue mechanism is even less understood compared with the deformation mechanism. Takahara et al. ${ }^{11}$ studied the fatigue mechanism of SPUU in terms of dynamic mechanical measurements and IRD and discussed the change of the degree of phase separation. We studied the fatigue mechanism of SPUU by using mechanical measurements, DSC, IRD, and proposed a three stage process of the fatigue mechanism ${ }^{6}$ : the domain orientation, the phase-mixing, and the segment orientation stages.

In this paper we focus attention on the structural change of SPUU due to fatigue and compare the mechanism with that of a uniaxial deformation based on structural analyses by means of SALS, SAXS, and IRD.

\section{EXPERIMENTAL}

\section{Samples}

Two types of SPUU consisting of poly(tetramethylene glycol) (PTMG), 4,4'diphenylmethane diisocyanate (MDI), and 1,2-propylene diamine (PDA) were used. The chemical structure of SPUU is shown in Chart I. These SPUUs have different numberaverage molecular weights $\left(\bar{M}_{n}\right)$ of PTMG. The samples with $\left(\bar{M}_{n}\right)$ equal to 856 and 1343 were designated as TM-1 and TM-3, respectively. The molar ratio of MDI, PTMG, and PDA was chosen to be $2: 1: 1$ both for TM-1 and TM-3. The numbers of the repeating units, $m$ and $n$, were assumed to be the same as those reported by Kimura et al. ${ }^{1}$ The weight fractions of the hard segment were 0.229 and
Chart I. Chemical structures of SPUU

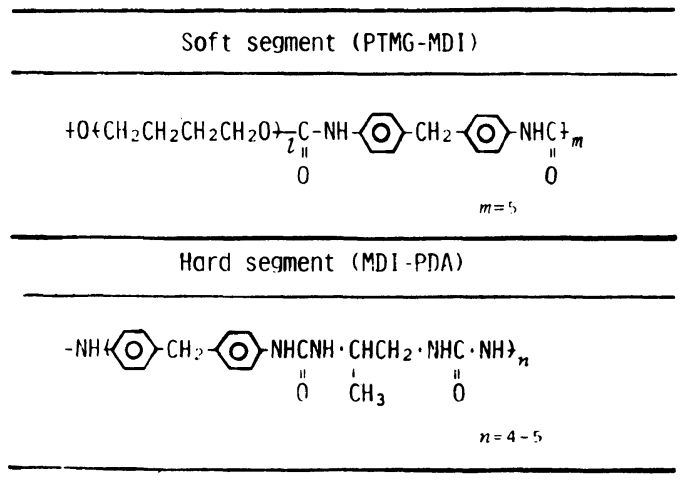

0.171, respectively for TM-1 and TM-3.

The samples dissolved in dimethyl formamide (DMF) were cast onto a glass plate at $60^{\circ} \mathrm{C}$ in the atmosphere of the solvent vapor. The dried sample was further dried in a vacuum oven kept at $60^{\circ} \mathrm{C}$ until use. The film thickness was about $200 \mu \mathrm{m}$.

\section{Fatigue Test}

The fatigue test was carried out by applying a $20 \%$ dynamic sinusoidal strain in addition to a $100 \%$ static strain at a rate of $10 \mathrm{~Hz}$. The details of the fatigue test are described in ref 6 . The fatigued sample code is designated by attaching the fatigue time in thousand seconds after the as-cast sample code as we employed in ref 6 .

\section{Small Angle X-Ray Scattering (SAXS)}

SAXS patterns were taken both photographically and photometrically as a function of the scattering and azimuthal angles. In the case of the photographic method, nickel filtered X-ray from a generator (Rigaku Denki, Cat. No. 4036A1) operated at $40 \mathrm{kV}$ and $27.5 \mathrm{~mA}$ was used as the incident beam. SAXS patterns were recorded on a X-ray film for 48 hours. On the other hand, the scattered intensity distributions were obtained with a $12 \mathrm{~kW}$ rotating anode $\mathrm{X}$-ray generator and a position sensitive proportional counter (PSPC). The data were corrected for air scat- 
tering, transmission, sample thickness, and slit smearing. The details of the apparatus and procedure of data analyses are described elsewhere. ${ }^{12,13}$

\section{Small Angle Light Scattering (SALS)}

The cross polarized (so called $H_{\mathrm{v}}$ ) light scattering patterns were used to investigate superstructures of SPUU. The light source was a Helium-Neon laser, having a wavelength of $632.8 \mathrm{~nm}$ in vacuum.

\section{RESULTS AND DISCUSSION}

\section{Microphase Structure of As-Cast Samples}

Figure 1 shows $H_{\mathrm{v}}$-SALS and SAXS pat- terns of as cast TM-1 and TM-3. This shows that both TM-1 and TM-3 have heterogeneous structures in the dimensions both of a few tens of nanometers and of a few microns. $\mathrm{P}$ and $\mathrm{A}$ indicate the directions of the polarizer and analyzer, respectively. The ring diffraction patterns in SAXS photographs reflect the long period of the randomly oriented lamellar microdomain structure consisting of the hard and soft segment domains. $H_{\mathrm{v}}$-SALS patterns indicate the existence of a spherulitic or a rod-like texture. If we assume that the SALS pattern indicates a spherulitic texture, the radius of the spherulite, $R_{0}$, can be estimated by the Stein's equation ${ }^{14}$;

$$
R_{0}=4.09\left[4 \pi \sin \left(\theta_{\mathrm{m}} / 2\right) / \Lambda\right]^{-1}
$$

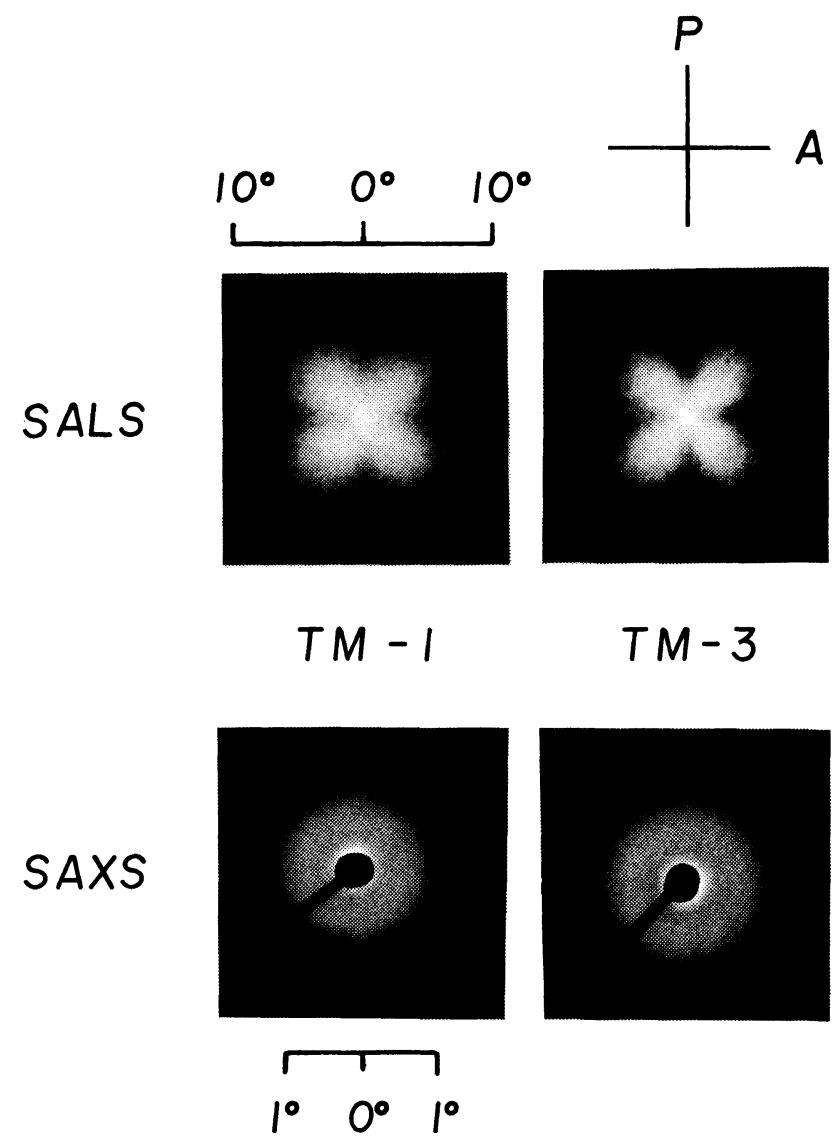

Figure 1. $H_{\mathrm{v}}$-SALS and SAXS patterns of as cast TM-1 and TM-3. P and A indicate the directions of the polarizer and analyzer. 
where $\left(\theta_{\mathrm{m}}\right)$ is the scattering angle at scattering intensity maximum and $\Lambda$ is the wavelength of the light in the medium. The estimated $R_{0}$ was about $3.0 \mu \mathrm{m}$ both for TM-1 and TM-3. The validity of the spherulite structure will be discussed later.

Figure 2 shows the desmeared SAXS intensity curves for TM-1 and TM-3. The scattering maximum indicates the microphase separated structure. Since the systems were isotropic, the intensity curves were multiplied with the so-called Lorentz factor, as shown in Figure 3. The long period $D$ estimated from Figure 3 was 9.2 and $9.4 \mathrm{~nm}$, respectively for TM-1 and TM-3. It is reasonable that TM-3 has a longer $D$ value, but the dif-

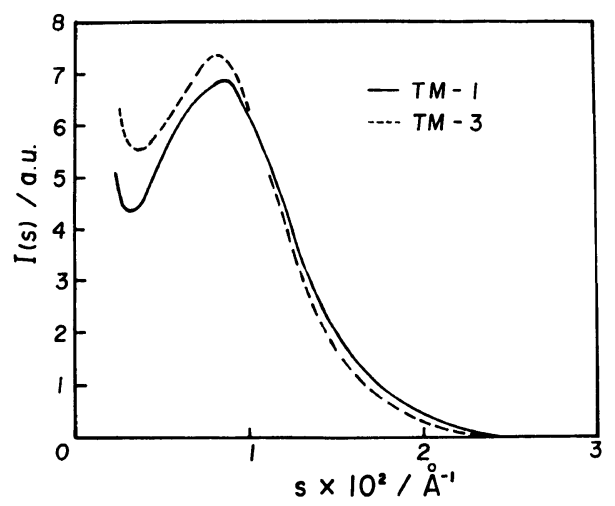

Figure 2. Desmeared SAXS intensity distributions of as cast TM-1 and TM-3.

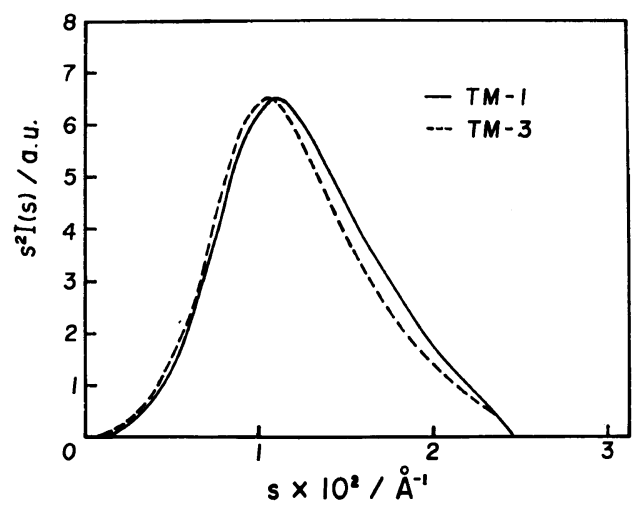

Figure 3. Lorentz corrected desmeared SAXS intensity distributions of as cast TM-1 and TM-3. ference is too small if one compares the soft segment molecular weights of TM-1 and TM-3. This means that the long spacing is not sensitive to the soft segment molecular weight when the sample is as cast. The poor soft segment molecular weight dependence of the long period is due to polydispersity of the molecular weight of the sample and to the poor organization of the phase separated structure. That is, the chain conformation was frozen during solidification, which gave rise to a lack of molecular characteristics in the microdomain sizes. This kind of phenomenon in block copolymers is discussed by Shibayama et al. ${ }^{15}$ as "non-equilibrium effects in phase separation."

The degree of phase separation can be estimated by the invariant. If the system is comparised of an ideal two-phase system, the invariant is given by

$$
Q=K \int_{0}^{\infty} s^{2} I(s) \mathrm{d} s=K^{\prime} \phi_{\mathrm{A}} \phi_{\mathrm{B}}(\Delta \rho)^{2}
$$

where $s$ is the magnitude of the scattering vector, $\phi_{\mathrm{A}}$ and $\phi_{\mathrm{B}}$ the volume fraction of $\mathrm{A}$ and $\mathrm{B}$ phases, and $K$ and $K^{\prime}$ are constants which are independent of samples. $(\Delta \rho)^{2}$ is the square of electron density difference between $A$ and $B$ and is given by

$$
(\Delta \rho)^{2}=\left(\rho_{\mathrm{A}}-\rho_{\mathrm{B}}\right)^{2}
$$

where the $\left(\rho_{i}\right)$ is the electron density of the $i$ phase. For convenience, the ratio of the invariants $\left(Q_{1} / Q_{3}\right)$ was used to compare the degree of phase separation between TM-1 and TM-3, where $Q_{i}$ is the invariant of the system $i$. The ratio was obtained as

$$
Q_{1} / Q_{3}=1.049
$$

By assuming the volume fraction equal to the weight fraction as a zero-th order approximation, one can get the ratio of the electron density difference,

$$
\Delta \rho_{1} / \Delta \rho_{3}=0.918
$$

This shows that the electron density difference 
between the hard segment-rich and the soft segment-rich domains of TM-1 is lower than that of TM-3, indicating that the degree of phase separation of TM-3 is higher than that of TM-1. A similar result was obtained from thermal analysis. ${ }^{4,6,16}$

The cohesive force index, ${ }^{11,17} \mathrm{Ch}$, obtained from an infrared absorption experiment also gives the degree of microphase separation. The cohesive force index is defined as the ratio of the relative absorbance of hydrogen bonded urea carbonyl absorption peak at $1640 \mathrm{~cm}^{-1}$ to that of the $\mathrm{C}=\mathrm{C}$ stretching absorption peak of the benzene ring at $1600 \mathrm{~cm}^{-1}$, where the latter is an internal reference. The cohesive force indices were 0.986 and 1.034 , respectively for TM-1 and TM-3, indicating again the higher degree of microphase separation of TM-3.

\section{Structural Changes due to Uniaxial Stretching}

Figure 4 shows SALS and SAXS patterns from the as cast and stretched films (upper) and the orientation behavior of the hard and soft segments as a function of $\%$-elongation (lower). The draw ratio is indicated by (a) to (d) in the figure of the orientation factor $v s . \%$ elongation. The orientation factor, $F_{i}^{\mathrm{D}}$, was determined by infrared dichroism, IRD. $F_{\mathrm{C}=\mathrm{O}}^{\mathrm{D}}$ and $F_{\mathrm{CH}_{\mathrm{H}}}^{\mathrm{D}}$ shown in Figure 4 denote the orientation factors derived from the urea carbonyl stretching band $v(\mathrm{C}=\mathrm{O})$ at $1640 \mathrm{~cm}^{-1}$ and the asymmetric methylene stretching band $v_{\mathrm{a}}\left(\mathrm{CH}_{2}\right)$ at $2940 \mathrm{~cm}^{-1}$, respectively. ${ }^{6}$ Therefore, $F_{\mathrm{C}=\mathrm{O}}^{\mathrm{D}}$ gives the orientation behavior of the hard segments, and $F_{\mathrm{CH}}^{\mathrm{D}}$ gives that of the soft segments. Since the direction of the transition moments of the $v(\mathrm{C}=\mathrm{O})$ and $v_{\mathrm{a}}\left(\mathrm{CH}_{2}\right)$ are nearly perpendicular to the chain axis, ${ }^{18} F^{\mathrm{D}}<0$ and $F^{\mathrm{D}}>0$ indicate the parallel and perpendicular orientations of the segments with respect to the stretching direction (so called positive and negative orientations, respectively). Though the soft segments are preferentially oriented along the stretching direction with increasing elongation, the hard segments are at first oriented negatively at relatively small elongation and then turn positively with further stretching. The negative orientation of the hard segments results from the long axis (lateral direction) of the lamellae being temporarily oriented to the stretching direction which is widely observed in SPUU ${ }^{1,5,6}$ and SPU., ${ }^{4,9}$

As shown in the SAXS patterns in Figure 4, a four point pattern can be observed up to about $200 \%$ elongation, indicating a herringbone structure. With increasing strain, this four point pattern becomes diffuse and is spread out toward the meridian, which suggests disintegration of lamellae and broadening of the interlamellar spacing distribution. Information on superstructures may be obtained from SALS analysis. With increasing elongation, SALS patterns are flatted perpendicular to the stretching direction, indicating deformation of a spherulitic texture or orientation of a rod-like texture toward to the stretching direction. Figure 4(e) shows SALS and SAXS patterns from a sample relaxed from $400 \%$ elongation. The SALS pattern indicates that the spherulitic or rod-like structure is partially restored. On the other hand, the SAXS pattern shows that diffuse scattering maxima move toward the stretching direction and shift to higher angle position compared with other patterns.

Based on the experimental results above, we discuss a possible structural unit responsibly for the structural change due to uniaxial stretching. Figure 5 shows the three models: the independent lamella (a), the rod (b), and the spherulite models (c). The thick straight lines and thin wavy lines schematically show the hard and the soft segments. This model does not exclude the possibility of folding of the hard segment as proposed by Koberstein and Stein. ${ }^{19}$ These are basically a two-phase models consisting of hard segment lamellae and soft segment matrix. The arrows indicate the movement of the structural unit when a uniaxial strain is applied along the stretching 


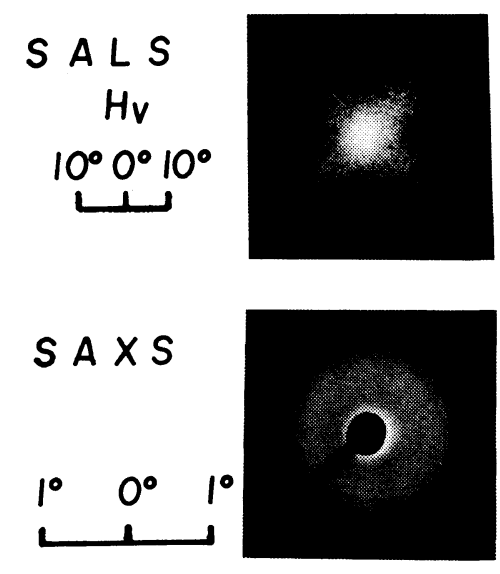

(a)
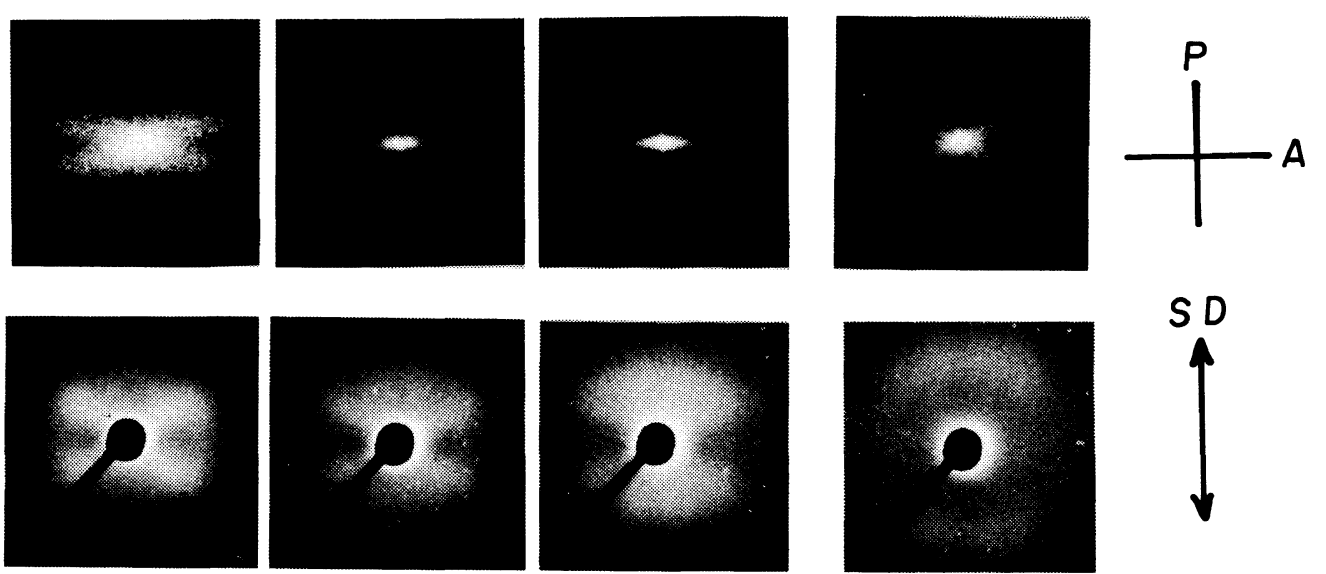

(b)

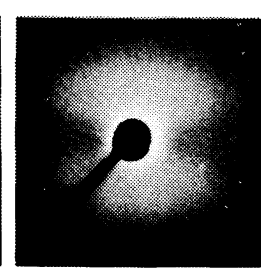

(d)

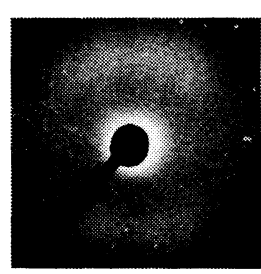

(e)

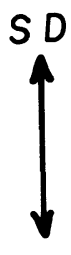

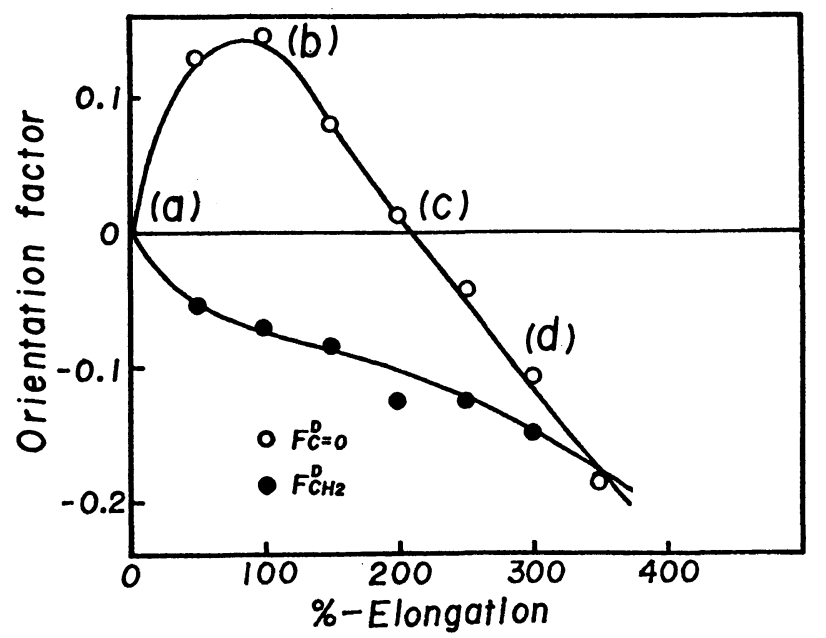

Figure 4. $H_{\mathrm{v}}$-SALS and SAXS patterns and orientation factors of $\mathrm{CH}_{2}$ and urea $\mathrm{C}=\mathrm{O}$ groups as functions of $\%$-elongation. $\mathrm{P}, \mathrm{A}$, and SD indicate the directions of the polarizer, the analyzer, and the elongation. 


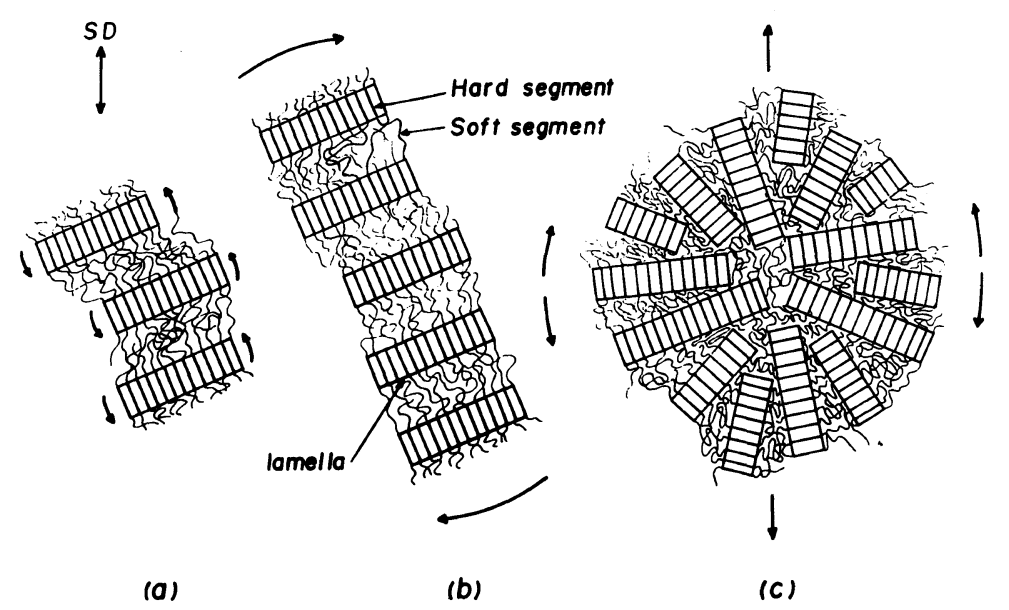

Figure 5. Three possible structural units of deformation: the independent lamella (a), the rod (b), and the spherulite models (c). The arrows indicate the direction of movement of the lamellae when an external strain is applied in the direction of SD.

direction (SD). The independent lamella model is the same as the Bonart's ${ }^{8,9}$ or Desper's model,${ }^{10}$ which is suitable to explain IRD and SAXS results: the intial negative orientation of the hard segment (IRD) and the four point pattern (SAXS) when the sample is stretched. This mechanism is true when individual lamellae are mechanically isolated from each other, i.e., floating in a fluid. However individual lamellae are not isolated but tied together by soft segment chains. Thereby each lamella must turn to the opposite direction from that indicated in Figure 5(a) when stretched. In addition one needs to introduce a superstructure, like a rod or a spherulite as shown in Figure 5(b) or (c) in order to explain the SALS results observed here and by Kimura et al. ${ }^{1,5}$ The SALS pattern favors a rod like structure rather than a spherulitic structure because it is difficult to detect a intensity maximum in the radial direction along the azimuthal angle of $45^{\circ} .{ }^{20}$ The rod model, however, cannot explain the negative orientation of the hard segment by stretching. Kimura et al. ${ }^{1}$ reported from their SALS and optical microscopic studies that SPUU, whose chain is extended with diaminodiphenyl methane or ethylenediamine, has a clear spherulite structure. They also pointed out the possibility of a similar structure in the SPUU extended with 1,2-propylene diamine which is the case studied here. Therefore, we adopt the spherulite texture as a model of deformation. The lack of peak maximum might be due to ill-organized spherulites having low content of hard segment domains and broadly distributed size. We assume here that the spherulite is volume filled but very sparse in order to simplify the discussion in the context of a two-phase model.

By employing a spherulitic structural unit, the deformation mechanism is explained as shown in Figure 6. According to the spherulite deformation models ${ }^{21-23}$ the deformed structure can be separated into three zones: the equatorial, diagonal, and polar zones as shown in Figure 6. The radius of the deformed spherulite at a given azimuthal angle $\Omega$ is given by

$$
R=R_{0} \frac{\lambda^{1 / 2}}{\left(\lambda^{-1} \cos ^{2} \Omega+\lambda^{2} \sin ^{2} \Omega\right)^{1 / 2}}
$$

where $\lambda$ is the draw ratio. On deformation, lamellae in the equatorial zone are splayed apart from each other and destroyed into fragments. On the contrary, those in the polar zone are mainly squeezed out. Lamellar domains along the direction where $R=R_{0}$ (the 


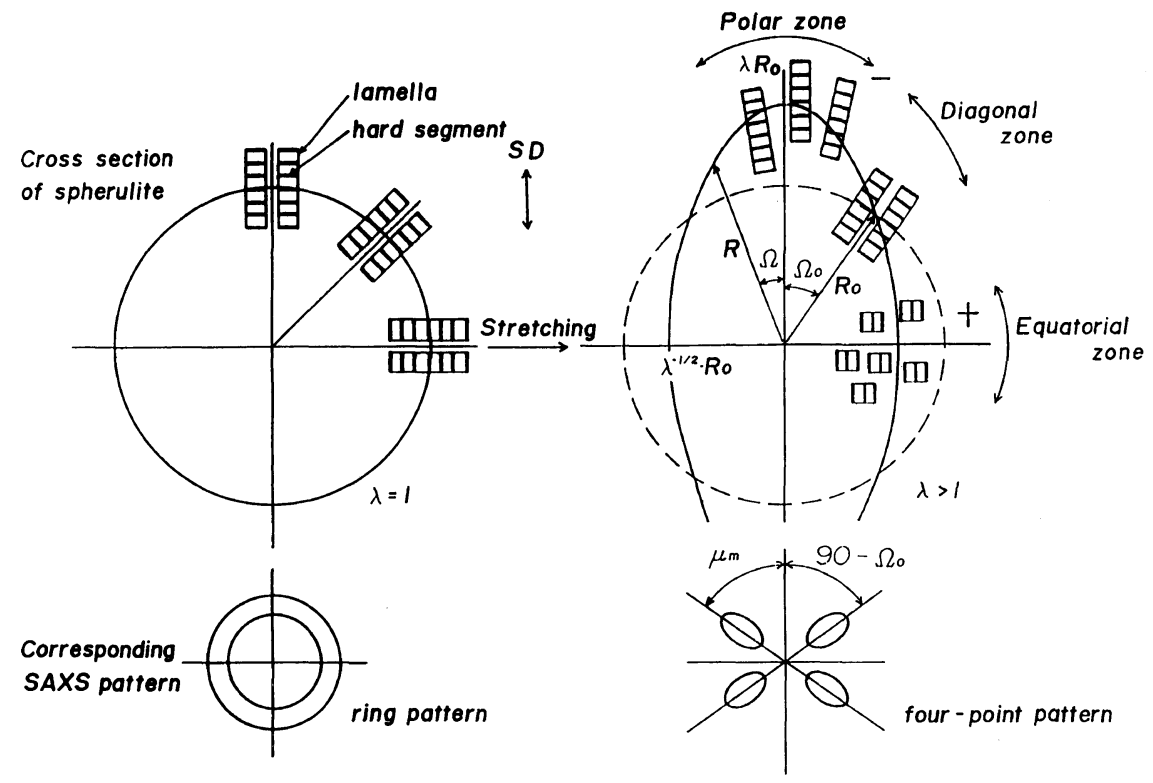

Figure 6. Schematic diagram showing the deformation mechanism of SPUU spherulite. The rectangles, squares, and the thick straight lines denote the hard segment lamellae, the fragmented domains, and the hard segments, respectively. + and - signs indicate the sign of the orientation factor of the hard segment located at the corresponding zero.

Table I. Characteristic azimuthal angles $\Omega_{0}, \Omega_{0}^{\prime}, \mu_{\mathrm{m}}$, and $\mu_{\mathrm{d}}$ of deformed spherulite as functions of the draw ratio $\lambda$

\begin{tabular}{|c|c|c|c|c|c|}
\hline \multirow{2}{*}{$\lambda$} & \multirow{2}{*}{$\%$-Elongation } & \multicolumn{2}{|c|}{ Under stretched } & \multicolumn{2}{|c|}{ After relaxed } \\
\hline & & $\left(90^{\circ}-\Omega_{0}\right)$ & $\mu_{\mathrm{m}}$ & $\left(90^{\circ}-\Omega_{0}^{\prime}\right)$ & $\mu_{\mathrm{d}}$ \\
\hline 2 & 100 & 49.1 & 55.0 & 22.2 & 27.0 \\
\hline 3 & 200 & 56.4 & 60.0 & 16.1 & - \\
\hline 4 & 300 & 60.8 & 65.0 & 12.6 & - \\
\hline
\end{tabular}

diagonal zone) are more or less preserved, and are responsible for the four point diffraction pattern. The azimuthal angles $\Omega_{0}$ and $\Omega_{0}^{\prime}$ where $R$ is equal to $R_{0}$ under stretched and after relaxed conditions, respectively, are easily obtained by

$$
\begin{aligned}
\Omega_{0}= & \cos ^{-1}\left[\lambda /\left(\lambda^{2}+\lambda+1\right)^{1 / 2}\right] \\
& (\text { under stretched) } \\
\Omega_{0}^{\prime}= & \cos ^{-1}\left[1 /\left(\lambda^{2}+\lambda+1\right)^{1 / 2}\right]
\end{aligned}
$$

(after relaxed)
The values of $\Omega_{0}$ and $\Omega_{0}^{\prime}$ as functions of $\lambda$ are listed in Table I. The peak angle $\mu_{\mathrm{m}}$, the azimuthal angle at intensity maximum, and the defect angle (which will be discussed later) obtained from SAXS is also listed in the table. The scattering maximum due to the lamellar long period should appear in the direction of the lamellar normal, i.e., $\left(90^{\circ}-\Omega_{0}\right)$, which is in good agreement with the peak angle $\mu_{\mathrm{m}}$, although the deformation model is rather simple. In addition, the polar zone is dominant at relatively low elongation, where the hard segments are preferentially oriented in the 


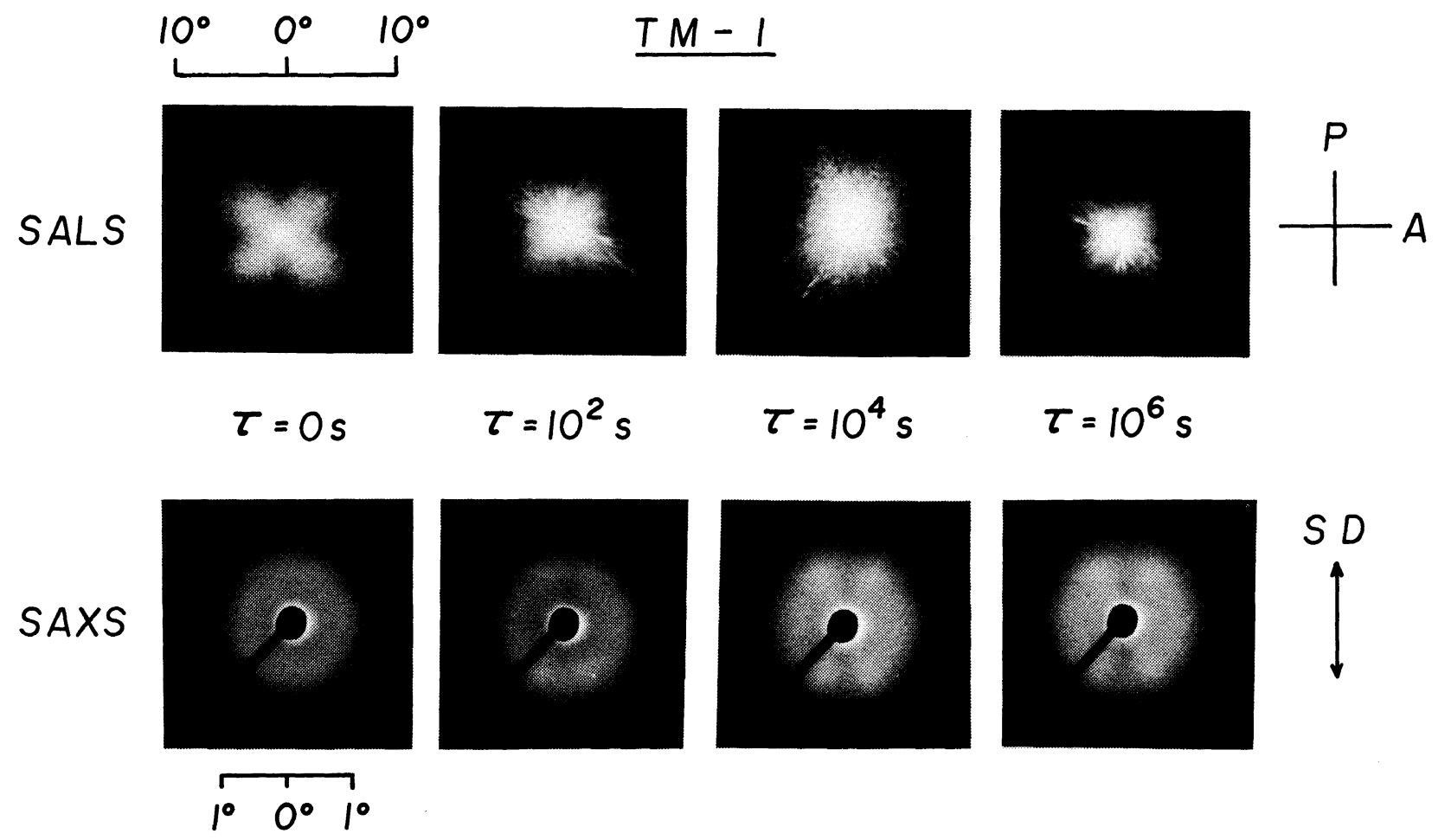

Figure 7. Variation of $H_{\mathrm{v}}$-SALS and SAXS patterns of TM-1 with time during fatigue, where $\tau$ denotes the fatigue time. 


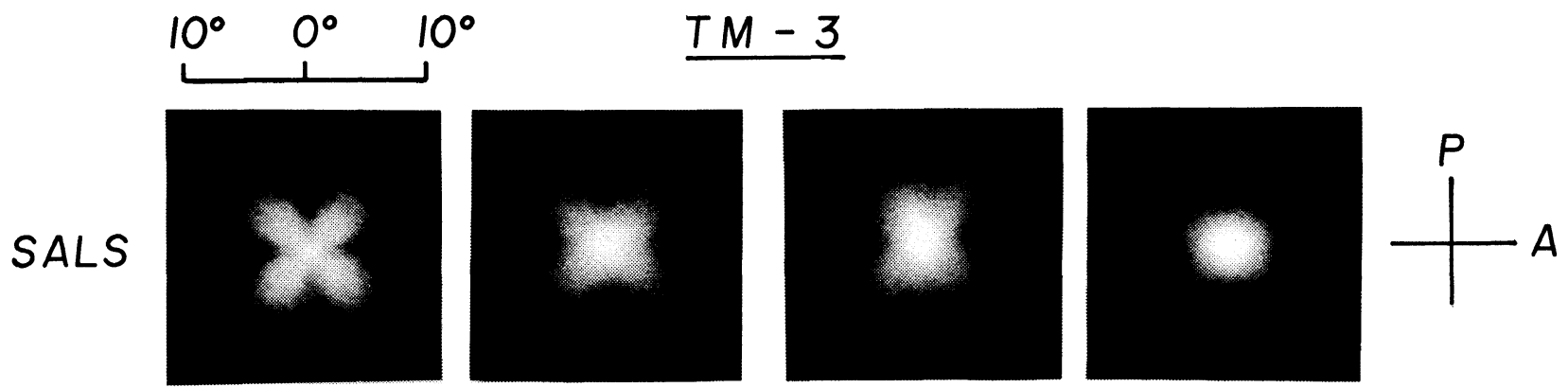

$$
\tau=0 \mathrm{~s} \quad \tau=10^{2} \mathrm{~s} \quad \tau=10^{4} \mathrm{~s} \quad \tau=10^{6} \mathrm{~s}
$$

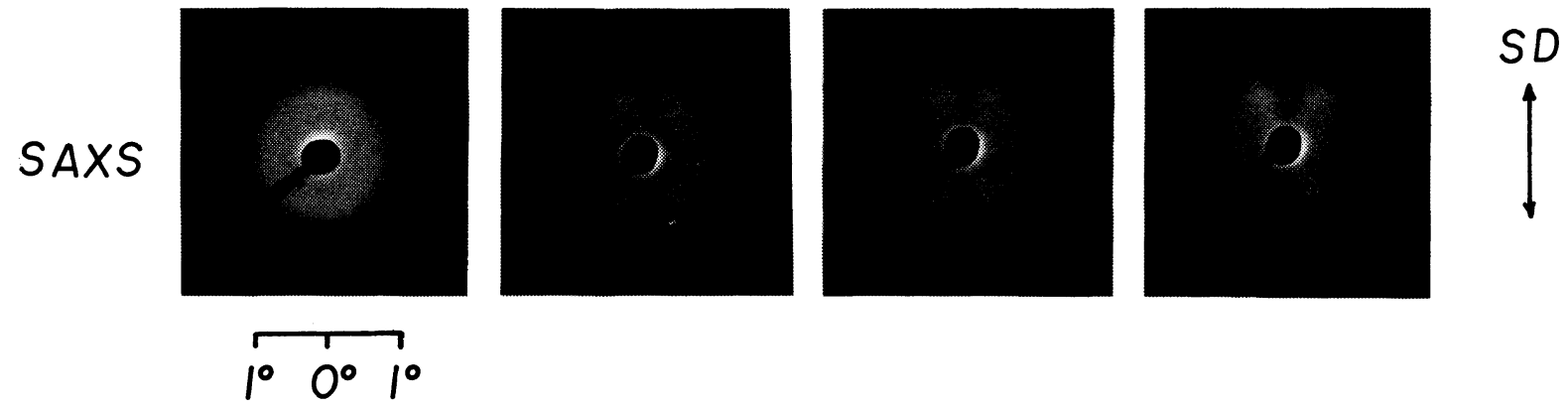

Figure 8. Variation of $H_{\mathrm{v}}$-SALS and SAXS patterns of TM-3 with time during fatigue process. 
tangential direction of the deformed spherulite, giving rise to a negative orientation as shown in Figure 4. With increasing elongation ( $\sim 300 \%$ ), the equatorial zone becomes dominant; that is, most of lamellae are disintegrated into fragments in which the hard segments orient positively. On relaxing the high stretching, the lamellar fragments are not reintegrated, and strain release takes place mainly in the soft segment chains and then the interfragment distance decreases.

\section{Structural Changes Due to Fatigue}

Figure 7 shows $H_{\mathrm{v}}$-SALS and SAXS photographs of the as cast and fatigued TM-1. These patterns were obtained from strain-free (relaxed) films. SALS photographs show flattening and then extinction of their characteristic four leaf patterns with fatigue time, indicating that the spherulitic structure is destroyed. On the other hand, the ring diffraction patterns in SAXS patterns have a characteristic defect in intensity along the meridian and local concentration of scattered intensity at the edge of the defect. We define "the defect angle $\mu_{\mathrm{d}}$ " as the angle between the meridian and the azimuthal angle at intensity maximum. The defect angle seems to become larger with fatigue time. Similar behavior was seen in TM3 as shown in Figure 8. The appearance of the defect angle and local concentration in intensity result from the same mechanism as discussed in Structural Changes Due to Uniaxial Stretching.

Figure 9 schematically summarizes the changes of SAXS patterns due either to uniaxial stretching (and release) or fatigue and release. The uniaxial stretching gives rise to a herringbone structure as characterized by the four point patterns discussed in Structural

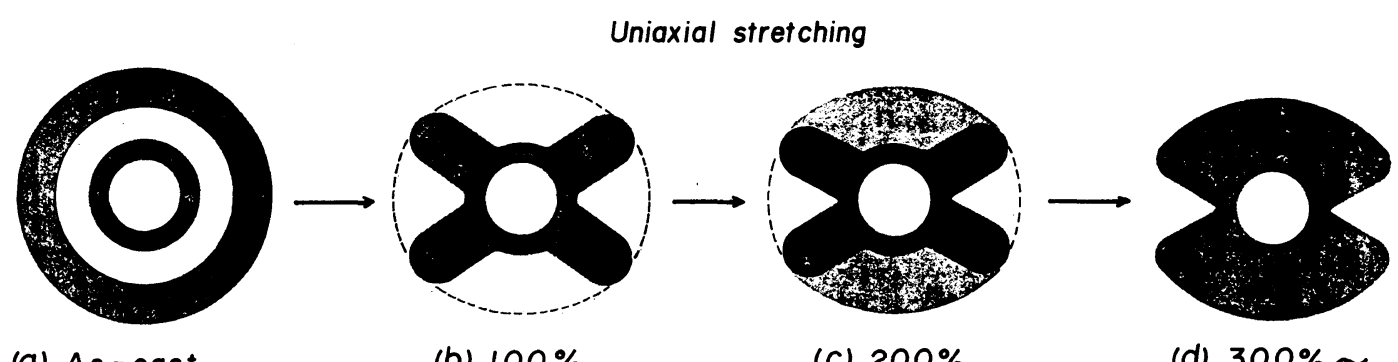

(a) As-cast

(b) $100 \%$

(c) $200 \%$

(d) $300 \% \sim$

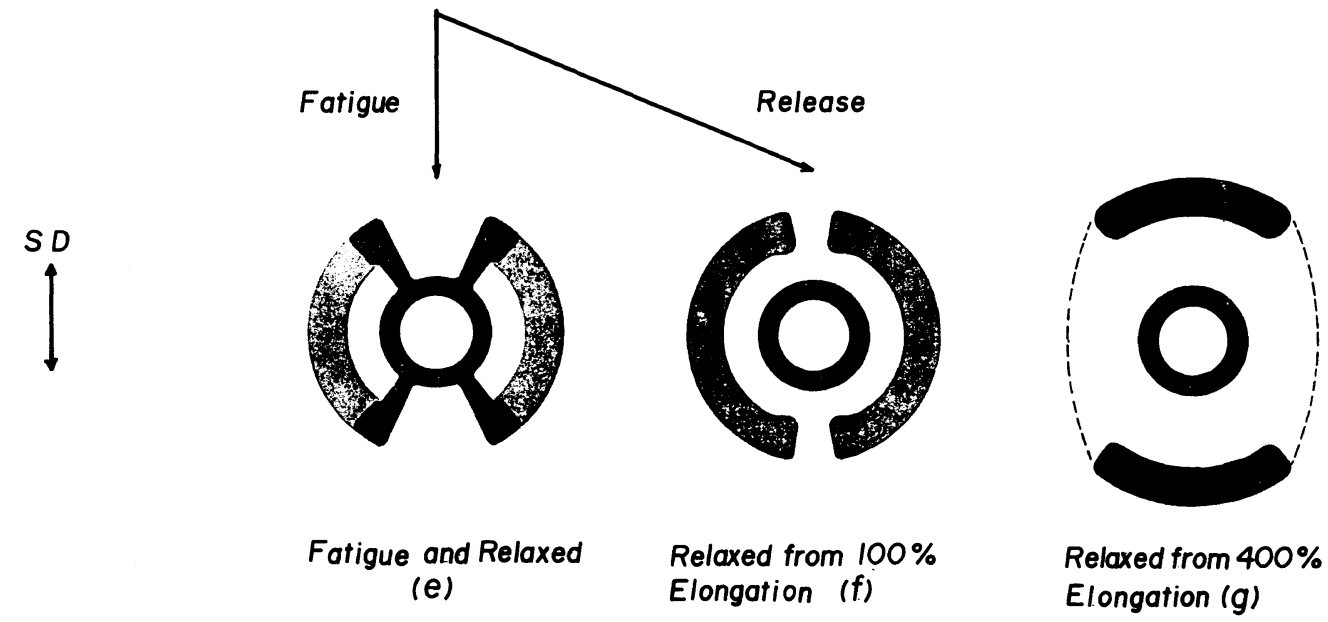

Figure 9. Schematic diagram showing the change of SAXS patterns due to uniaxial elongation or fatigue and release. 
Changes Due to Uniaxial Stretching. Similar behavior has often been observed in uniaxial stretching of semicrystalline polymers ${ }^{24}$ and block copolymers having lamellar microdomains. $^{25}$ These four point patterns are transformed to two point patterns with increasing stretching in which fragmented domains are oriented along the stretching direction. By relaxing the film, the two point patterns moved to higher scattering angle. This indicates shortening of the interdomain distance which is shorter than the original one. The lamellar domain structure was not restored by relaxing from $400 \%$ stretching. Since the fatigue experiments were performed by applying $100 \%$-static strain as an offset, the defect around the meridian emerged by the first stretch and release. The defect angle becomes bigger with increasing fatigue time. The lamellar domain structure is more or less preserved during fatigue except in the equatorial zone of the spherulite. Fragmentation of the lamellar structure around the equator is responsible for the defect of the diffraction maximum around the meridian.

Figure 10 shows the defect angle $\mu_{\mathrm{d}}$, the residual strain, the orientation factor of the urea carbonyl group $F_{\mathrm{C}=\mathrm{O}}^{\mathrm{D}}$, and the cohesive force index, $\mathrm{Ch}$, for TM-1 and TM-3 as a function of the logarithm of the fatigue time, $\log \tau$. The dotted lines divide the fatigue process of the TM-1 into three stages; i.e., the domain orientation (Stage I), the phase mixing (Stage II), and the segment orientation (Stage III) as we proposed in ref 6. In the case of TM-1, the defect angle remains constant in Stages I and II, which is about $27^{\circ}$, then increases in Stage III. The residual strain goes up monotonously from Stage I. On the other hand, the hard segment orientation has a characteristic feature as a function of fatigue time corresponding to the

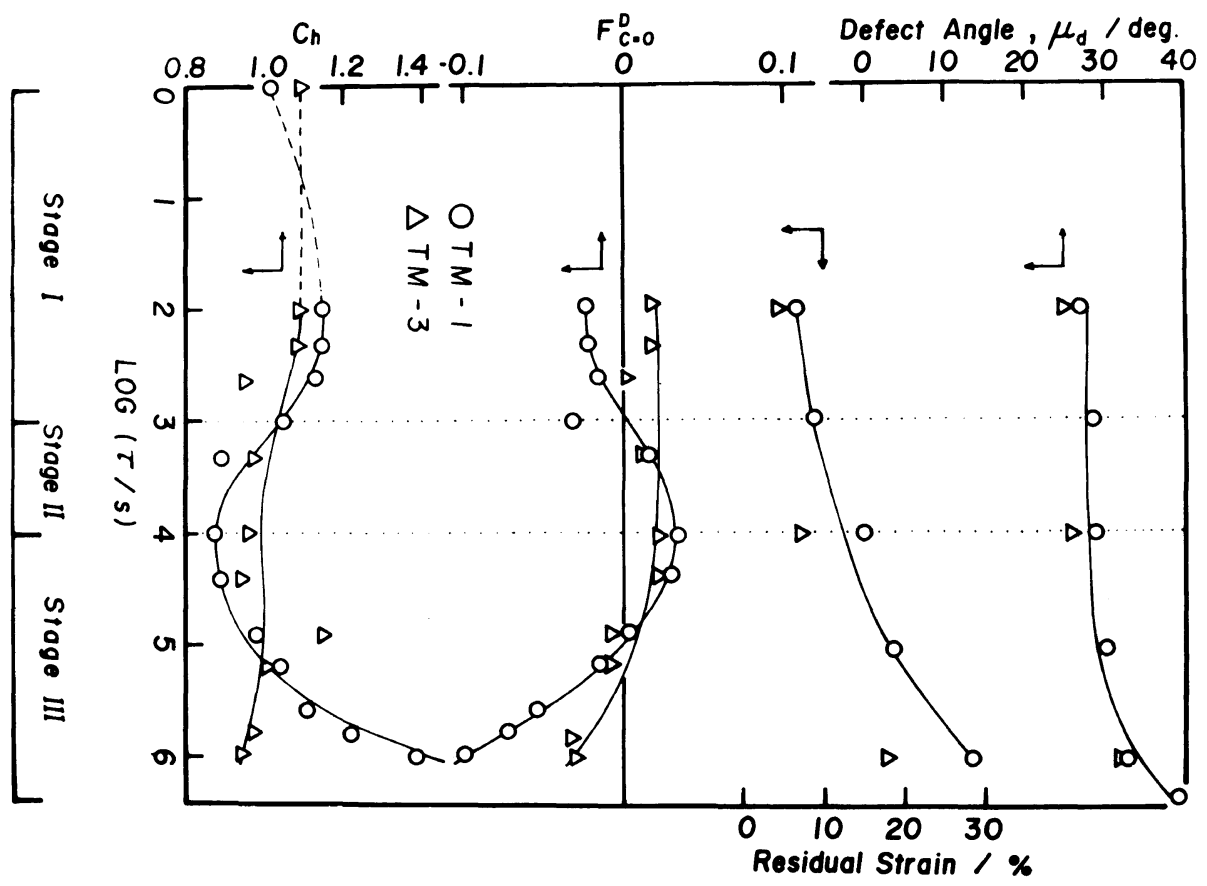

Figure 10. Changes of the defect angle, $\mu_{\mathrm{d}}$, the residual strain, the orientation factor of the urea carbonyl group of the hard segments, $F_{\mathrm{C}=\mathrm{O}}^{\mathrm{D}}$, and the cohesive force index, $\mathrm{Ch}$, for TM-1 and TM-3 as functions of the logarithm of the fatigue time. Negative $F_{\mathrm{C}=\mathrm{O}}^{\mathrm{D}}$ means positive orientation of the hard segments. The fatigue stages of TM-1 are indicated at the bottom. 


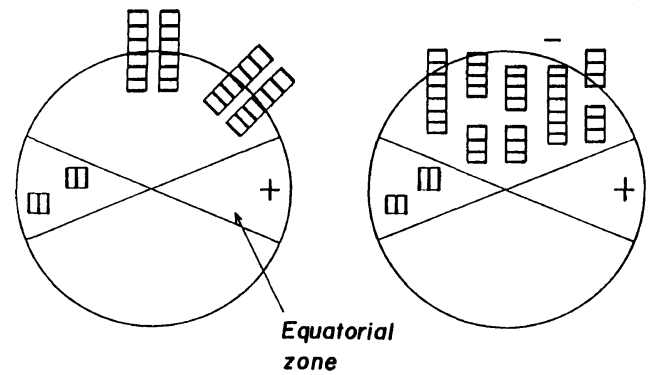

Stage I

Stage II

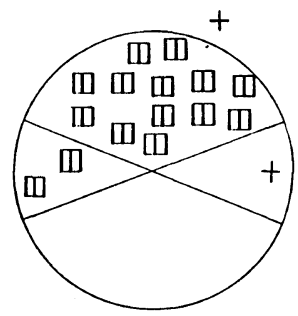

Stage III

Figure 11. Schematic diagram showing the fatigue mechanism of SPUU; Stage (I), Stage (II), and Stage (III). Meaning of the symbols and signs is the same as in Figure 6.

change of $\mathrm{Ch}$ as we discussed in ref 6 . It tends to strong positive orientation after $\tau=$ $10^{4} \mathrm{~s}$. The cohesive force index keeps a constant value up to $\tau=10^{3} \mathrm{~s}$, then decreases until $\tau=10^{4} \mathrm{~s}$ and then steeply increases. In the case of TM-3, the steep increase of the cohesive force index is not seen in this time scale, suggesting that TM-3 is less sensitive to fatigue than TM-1. This is due to the longer soft segment of TM-3. That is, TM-3 is better phase separated as discussed in Microphase Structure of As-Cast Samples and the longer soft segment reduces plastic deformation of the hard segments during the fatigue test.

Based on the experimental results discussed above, we propose a structural change model due to fatigue as shown in Figure 11. After the first cycle of the fatigue process the equatorial zone is destroyed whereas the diagonal and polar zones are restored. The diagonal zone is characterized by the azimuthal angle $\Omega_{0}^{\prime}$ or the defect angle $\mu_{\mathrm{d}}$. As shown in Table I and in Figure $10, \Omega_{0}^{\prime}$ and $\mu_{\mathrm{d}}$ are $67.8^{\circ}$ and $27.0^{\circ}$, respectively. Therefore $\left(90^{\circ}-\Omega_{0}^{\prime}\right)$ is close to $\mu_{\mathrm{d}}$, indicating again good accordance between the experimental results and the prediction based on the deformation model. At Stage I, most of the lamellae orient and reorient due to repetitive stimuli without significant destruction and only the lamellae in the equatorial zone are destroyed into fragments when stretched and an overshooting of strain relaxing occurs when relaxed. Both these lead to positive orientation as shown in Figure 10. The contribution of the fragmentation at the equatorial zone is rather small and does not affect $\mathrm{Ch}$. At Stage II, due to repetitive stimuli, the polar zone suffers non-reversible doformation; in this case, permanent lamellar orientation and fragmentation (the phase mixing). This contribution becomes bigger than that of the fragmentation at the equatorial zone resulting in the gross negative orientation at this stage. The increase of fragmentation gives the decrease of Ch. At Stage III, fragmented small domains tend to orient with those segments parallel to the stretching direction (the segment orientation). Finally, rearrangement of the small fragments takes place, leading to rapid increase of $\mathrm{Ch}$ at the end of Stage III. Changes of the defect angle and residual strain qualitatively explain this fatigue process. This is consistent with the conclusion in ref 6 where we discussed the results of hard and soft segment orientations, the cohesive force index, and thermal and mechanical properties. It should be noted, however, that the proposed mechanism is only valid in the case of the fatigue test employed here. Larger static strain 
may lead to phase mixing from the beginning and the fatigue process starts from Stages I and II at the same time.

\section{CONCLUSIONS}

The fatigue mechanism of segmented poly(urethaneureas) TM-1 and TM-3 was investigated. The cross polarized small angle light scattering of the as cast original films showed four leaf patterns indicating the existence of a spherulitic texture in both TM-1 and TM-3. The negative orientation followed by the positive orientation of the hard segment due to uniaxial stretching is explained, by a spherulite deformation model, i.e., the lamellar orientation followed by the segment orientation. The structural change model of segmented poly(urethaneureas) is thus proposed based on spherulite texture. SAXS patterns showed that selective destruction around the eqator of a spherulite occurs due to fatigue. As we discussed in ref 6 , the fatigue process consists of three stages: the domain orientation, the phase mixing, and the segment orientation stages. It was also found that the longer soft segments delayed the onset of Stage III.

Acknowledgments. We acknowledge partial financial support of the Ministry of Education, Science, and Culture of Japan, under Grant 61750842. We are grateful to Dr. T. Matsuda, National Cardivascular Center Research Institute, Suita, Osaka, for kindly supplying the samples. Thanks are also due to Prof. T. Hashimoto, Department of Polymer Chemistry, Kyoto University, for the SAXS apparatus.

\section{REFERENCES}

1. I. Kimura, H. Ishihara, H. Ono, N. Yoshihara, S. Nomura, and H. Kawai, Macromolecules, 7, 355 (1974); H. Ono and I. Kimura, Kagakuzoukan, 50, 181 (1971)

2. C. S. P. Sung, T. W. Smith, and N. H. Sung, Macromolecules, 13, 117 (1980).

3. G. L. Wilkes and S. Abouzahr, Macromolecules, 14, 456 (1981).

4. C. B. Wang and S. L. Cooper, Macromolecules, 16, 775 (1983).

5. H. Ishihara, I. Kimura, and N. Yoshihara, J. Macromol. Sci. Phys., B22, 713 (1983-1984).

6. M. Shibayama, T. Kawauchi, T. Kotani, S. Nomura, and T. Matsuda, Polym. J., 18, 725 (1986).

7. J. A. Miller, S. L. Cooper, C. C. Han, and G. Pruckmayr, Macromolecules, 17, 1063 (1984).

8. R. Bonart, J. Macromol. Sci. Phys., 132, 115 (1968).

9. K. Hoffmann and R. Bonart, Makromol. Chem., 184, 1529 (1983).

10. C. R. Desper, N. S. Schneider, J. P. Jasinski, and J. S. Lin, Macromolecules, 18, 2755 (1985).

11. A. Takahara, J. Tashita, T. Kajiyama, and M. Takayanagi, Kobunshi Ronbunshu, 39, 203 (1982).

12. T. Hashimoto, S. Suehiro, M. Shibayama, K. Saijo, and H. Kawai, Polym. J., 13, 501 (1980).

13. M. Fujimura, T. Hashimoto, and H. Kawai, Mem. Fac. Eng., Kyoto Univ., 43(2), 224 (1981).

14. R. S. Stein and M. B. Rhode, J. Appl. Phys., 31, 1873 (1960).

15. M. Shibayama, T. Hashimoto, and H. Kawai, Macromolecules, '16, 1434 (1983).

16. C. S. Sung, D. B. Hu, and C. S. Wu, Macromolecules, 13, 111 (1980).

17. A. Takahara, J. Tashita, T. Kajiyama, and $\mathbf{M}$. Takayanagi, Polym. Prepr. Jpn., 32, No. 8, 2007 (1983).

18. G. M. Estes, R. W. Seymour, and S. L. Cooper, Macromolecules, 4, 452 (1975).

19. J. T. Koberstein and R. S. Stein, J. Polym. Sci., Polym. Phys. Ed., 21, 1439 (1983).

20. T. Hashimoto, H. Kawasaki, and H. Kawai, J. Polym. Sci., Polym. Phys. Ed., 16, 271 (1978).

21. K. Sasaguri, R. Yamada, and R. S. Stein, J. Appl. Phys., 35, 3188 (1964).

22. T. Oda, S. Nomura, and H. Kawai, J. Polym. Sci., A, 3, 1993 (1965).

23. K. Fujita, S. Suehiro, S. Nomura, and H. Kawai, Polym. J., 14, 545 (1982).

24. See, for example, A. Peterlin and G. Meinel, Makromol. Chem., 142, 227 (1971).

25. T. Pakula, K. Saijo, H. Kawai, and T. Hashimoto, Macromolecules, 18, 1294 (1985). 